\title{
In Search of Cosmic-Ray Antinuclei from Dark Matter with the GAPS Experiment
}

\section{Mengjiao Xiao ${ }^{a, *}$ on behalf of the GAPS Collaboration}

(a complete list of authors can be found at the end of the proceedings)

\author{
${ }^{a}$ Massachusetts Institute of Technology, Department of Physics, \\ 77 Massachusetts Ave, Cambridge, U.S.A \\ E-mail: mjxiao@mit.edu
}

The General Antiparticle Spectrometer (GAPS) is the first experiment optimized to identify lowenergy $(\lesssim 0.25 \mathrm{GeV} / n)$ cosmic antinuclei, in particular antideuterons from dark matter annihilation or decay. The GAPS program will deliver unprecedented sensitivity to cosmic antideuterons, an essentially background-free signature of various dark matter models, as well as a high-statistics antiproton spectrum in the unexplored low-energy range, and leading sensitivity to cosmic antihelium. GAPS is currently under construction. The first Antarctic balloon flight of GAPS is planned for late 2022, and two additional flights are planned for the coming years. Based on measurements of our custom-developed instrument technology, including large-area lithiumdrifted silicon $(\mathrm{Si}(\mathrm{Li}))$ detectors and a large-acceptance time-of-flight system, as well as detailed instrument simulation and reconstruction studies, we present here the anticipated impact of the GAPS program on dark matter searches. This contribution discusses the current status of cosmic antinuclei studies while focusing on the science potential of GAPS.

$37^{\text {th }}$ International Cosmic Ray Conference (ICRC 2021)

July 12 th - 23rd, 2021

Online - Berlin, Germany

\footnotetext{
*Presenter
} 


\section{Introduction}

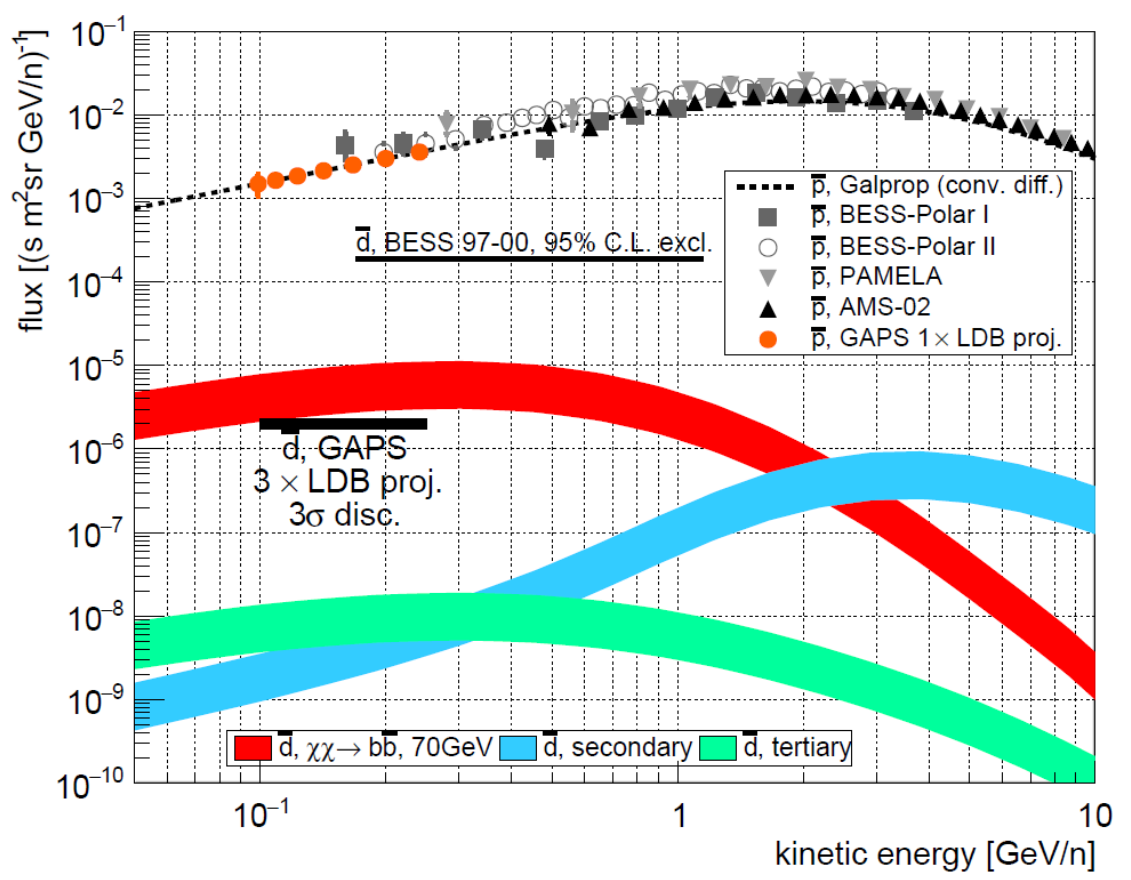

Figure 1: The GAPS antideuteron sensitivity ${ }^{[1][2]}$ (black bar) overlaid with current best upper limit by BESS $^{[3]}$, the predicted antideuteron flux from viable DM models ${ }^{[4]}$ (cyan), and antideutron fluxes from the secondary (blue) and tertiary (pink) astrophysical background. Orange points are GAPS projected antiproton measurement for one flight and dashed black line is the antiproton flux predication from Galprop model.)

The origin of dark matter (DM) is a driving question of modern physics. Finding dark matter in the laboratory and elucidating its properties could revolutionize our understanding of the fundamental building blocks of the universe. Dark matter experiments are usually categorized into collider searches, direct detection and indirect detection. Indirect dark matter detection is looking for Standard Model particles from dark matter annihilation or decay, such as high-energy photons or neutrinos, or excess antimatter. Cosmic rays are widely used for indirect dark matter detection with the assumption that cosmic rays from dark matter annihilation or decay follow different kinematics than those produced via conventional astrophysical processes. The common challenge for such experiments is the understanding of the astrophysical backgrounds. Compared to other cosmic rays, cosmic antinuclei provide an excellent channel for dark matter searches because of their relatively low level of production from conventional astrophysical processes ${ }^{[1]}$.

The General Antiparticle Spectrometer (GAPS), an Antarctic long-duration balloon (LDB) experiment, is the first experiment optimized to search for low-energy (kinetic energy $\lesssim 0.25 \mathrm{GeV} / n$ ) cosmic antideuterons, as well as measuring a high-statistics antiproton spectrum in an unexplored low-energy range. GAPS also provides a leading sensitivity to low-energy cosmic antihelium. The unique strength of the GAPS low-energy antideuteron search for DM lies in this channel's ultralow astrophysical background. Secondary or tertiary (astrophysical background) antideuterons are produced by the interaction of cosmic-ray protons or antiprotons with the interstellar medium. As illustrated in Fig. 1, the combination of the high production threshold for antideuterons, relativistic 


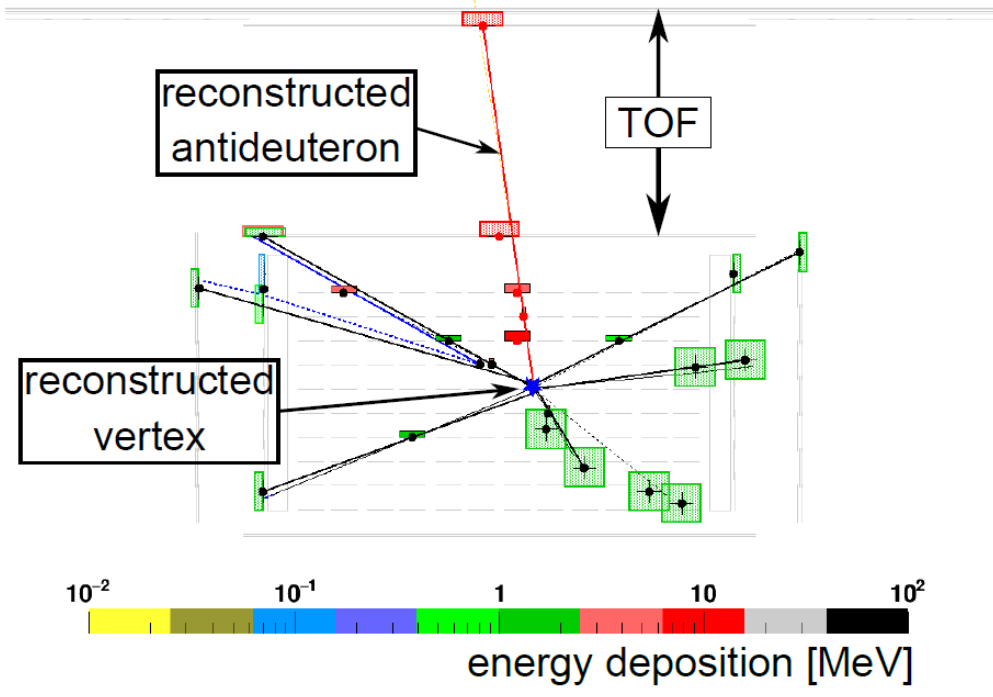

Figure 2: Antideuteron event topology in the GAPS instrument: the red line is the reconstruction of the primary antideuteron and the black lines reconstruct the secondary tracks from the stopping vertex inside the tracker. The colored boxes represent energy depositions in sensitive detector volumes: the largest of which (red) are on the primary track, and the green ones are on the secondary tracks. The detector materials (TOF system and ten silicon tracker layers) are in light gray.

kinematics, and the low abundance of high-energy cosmic rays, leads to a secondary or tertiary antideuteron flux that, compared to realistic DM models, is highly-suppressed and also boosted towards higher energies. In this scenario, low-energy antideuterons have long been recognized as just such a background-free, "smoking gun" DM signature ${ }^{[1]}$.

\section{Antinuclei Identification with the Exotic Atom Technique}

GAPS uses a novel detection technique based on exotic atom capture and decay to identify cosmic antinuclei ${ }^{[5]}$. As illustrated in Fig. 2, the time-of-flight (TOF) system measures the velocity, angle, and energy deposition $(\mathrm{d} E / \mathrm{d} x)$ of low-energy antinuclei when they pass through two layers of plastic scintillator. The TOF system also provides the high-speed trigger to the experiment. The incoming antiparticle is further slowed by energy losses in the $\mathrm{Si}(\mathrm{Li})$ tracker layers, and it eventually stops inside the tracker volume. The captured antiparticle then replaces a shell electron of the silicon to form an exotic atom in an excited state. This exotic atom de-excites and emits $\mathrm{X}$-rays promptly. Finally, the antiparticle annihilates with the silicon nucleus, producing a nuclear star of pions and protons. Both the produced X-rays and charged particles (protons and charged pions) are further detected by the $\mathrm{Si}(\mathrm{Li})$ tracker.

The simultaneous detection of the incoming particle by the TOF and the annihilation products by the tracker provides a powerful way to distinguish antinuclei from particles such as muons, protons, electrons or alphas. Furthermore, benefitting from its novel detection technique, GAPS provides multiple ways to discriminate between antiprotons and antideuterons: 1) The TOF measures the velocity of the incoming particle. Compared to antiprotons with the same velocity, antideuterons 


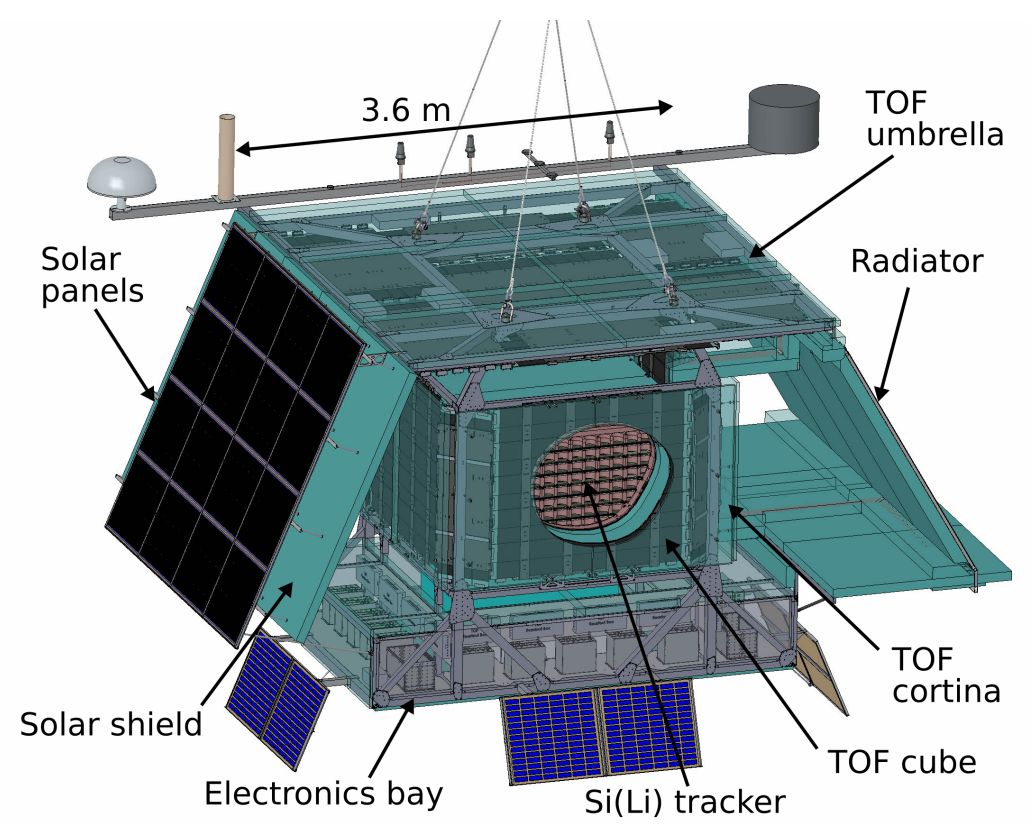

Figure 3: GAPS instrument design. The front panel of the cortina has been removed to show the inner detector and that the inner TOF has been cut open to show the tracker.

pass through more tracker layers and deposit more total energy. 2) After annihilation, antideuterons yield more charged particles (protons and charged pions) than antiprotons since antideuterons are more massive. 3) For a given target material, the energies of X-rays from the exotic atom are uniquely determined by the mass of the captured antinuclei, so the characteristic X-ray lines provide a further method for antinuclei identification. Combining the discrimination powers of the stopping depth, energy deposition profile, multiplicity of charged particles, and characteristic X-rays, the GAPS antinuclei mis-identification can be suppressed to below $10^{-6}$. Hence, the observation of one antideuteron in GAPS is sufficient to claim a discovery.

Based on the aforementioned detection principle, Fig. 3 shows the GAPS instrumental design, see S. Quinn's contribution for more details. The heart of the GAPS detector is a $1.6 \mathrm{~m} \times 1.6 \mathrm{~m} \times 1.0 \mathrm{~m}$ $\mathrm{Si}(\mathrm{Li})$ tracker. It measures the $\mathrm{d} E / \mathrm{d} x$ of the incoming particles, tracks the annihilation products (X-rays and charged particles), and reconstructs the annihilation vertex (combined with the TOF). The GAPS tracker is built with ten layers of custom-made large-area $\mathrm{Si}(\mathrm{Li})$ detectors ${ }^{[6][7][8]}$, and each layer covers an area of over $1 \mathrm{~m}^{2}$. The tracker is surrounded by a plastic scintillator TOF system. The GAPS TOF system consists of an inner "cube", top "umbrella" and four sides, called "cortina". The separation is $\approx 90 \mathrm{~cm}$ between the cube and the umbrella, and $\approx 30 \mathrm{~cm}$ between the cube and the cortina. The TOF is composed of plastic scintillator paddles with 6 SiPMs mounted at each end. The GAPS thermal system utilizes an oscillating heat pipe (OHP), which faces away from the Sun to maintain the instrument temperature ${ }^{[9]}$. The heat produced by the tracker will be transferred into space via a radiator. On the Sun-facing side, there is a solar array to supply power. The electronics bay houses the electronics and other mechanical supports. The full payload of the GAPS instrument is $\sim 2.5 \mathrm{t}$ and the total power consumption is $\sim 1.3 \mathrm{~kW}$. The instrument will serve for a series of long-duration balloon flights, each lasting around 35 days. 


\section{GAPS Scientific Potential}

GAPS will start the initial Antarctic LDB flight in late 2022 and plans two follow-up flights. The science objectives of the GAPS Antarctic flight program are as follows:

- Flight 1 will validate the novel antinuclei identification technique, start a new era of precision measurements of low-energy cosmic antiprotons, and deliver a leading sensitivity to cosmic antideuterons that will begin to probe viable DM models.

- Flights 2 and 3 will open sensitivity to more generic dark matter models with antideuterons.

- All flights will provide leading sensitivity to low-energy cosmic antihelium.

Using a Geant $4^{[10][11]}$-based simulation of the full GAPS instrument, the projections of sensitivities to antideuteron and antihelium- $3^{[12]}$, and antiproton flux were determined. The GAPS scientific potential is presented in the following.

\subsection{Antiproton Flux}

Previous antiproton measurements have been used to constrain DM models, as well as astrophysical production and propagation scenarios. Recent studies of the AMS-02 low-energy antiproton spectrum indicate a possible excess that could be consistent with $20-80 \mathrm{GeV}$ dark matter, invoking some of the same models indicated by the Galactic Center $\gamma$-ray excess ${ }^{[13]}$. However the DM interpretation is inconclusive due to astrophysical uncertainties. In addition, any antideuteron signal needs to be compatible with antiproton constraints. Hence, precision measurements of antiprotons are vital input for DM searches with antideuterons.

Fig. 1 illustrates the expected antiproton measurement at Top of the Atmosphere (TOA) for a GAPS 35-day Antarctic flight at $37 \mathrm{~km}$ float altitude; see more details in F. Rogers's contribution. GAPS extends the range of precision antiproton measurements about an order of magnitude lower in kinetic energy compared to the AMS-02 experiment. It needs to be noted that the current highestprecision AMS-02 antiproton data have an uncertainty of about $30 \%$ at $0.5 \mathrm{GeV} / n$. The crucial low-energy range $(\leq 0.25 \mathrm{GeV} / n)$ that the GAPS experiment is focused on is relatively unconstrained. The GAPS precision measurement of antiprotons in this unexplored low-energy range will include around two orders of magnitude more low-energy antiprotons than BESS ${ }^{[14]}$ and PAMELA ${ }^{[15]}$ combined. These measurements will provide strong constraints on DM candidates with masses $<30 \mathrm{GeV}$, and on primordial black hole evaporation on Galactic length scales. In addition, it will also offer detailed constraints of phenomena such as solar modulation that affect the low-energy antiproton flux.

\subsection{Antideuteron Science}

Cosmic antideuterons have not yet been observed, and any detection would open a new field of cosmic research. A broad range of DM models predict a low-energy antideuteron flux from DM annihilation or decay orders of magnitude above this astrophysical background. As illustrated in Fig. 1, the flux from a generic 70-GeV WIMP annihilating into antideuterons via $b \bar{b}$-pairs exceeds the standard astrophysical background by three orders of magnitude in the energy range below a few $\mathrm{GeV} / n$, even taking into account constraints from $\gamma$-ray and antiproton measurements ${ }^{[4]}$.

Fig. 4 illustrates the GAPS sensitivity to low-energy cosmic antideuteron searches with three LDB flights compared with antideuteron fluxes predicted by several specific DM models. These 


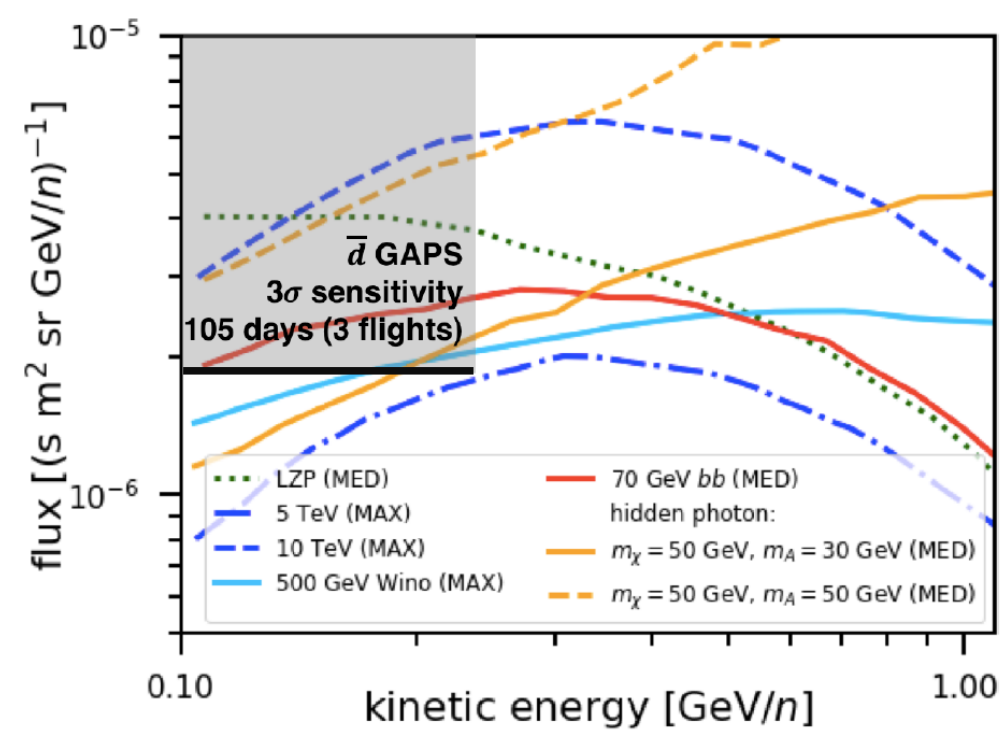

Figure 4: GAPS antideuteron sensitivity ( $3 \sigma$ discovery for 1 detected event) for three LDB flights, in comparison with viable DM models ${ }^{[4][18][21][22][23]}$

DM models include a right-handed Kaluza-Klein neutrino of extra-dimensional grand unified theories $(\mathrm{LZP})^{[16]}$, a decaying LSP gravitino ${ }^{[17]}$, a particle with gluonic decay channels ${ }^{[18]}$, and candidates in the next-to-minimal supersymmetric model (NMSSM) ${ }^{[19]}$. For a $3 \sigma$ discovery of one antideuteron event, the GAPS sensitivity will reach well below the thermal relic cross section for DM with masses below $20-100 \mathrm{GeV}$ annihilating into light quarks ${ }^{[20]}$. In contrast to direct detection and collider DM searches, the GAPS antideuteron search is also sensitive to certain heavy DM models, for example, (5-20 TeV) WIMP models ${ }^{[21]}$ and TeV-scale pure-Wino dark matter with a Sommerfeld enhancement mechanism ${ }^{[22]}$. Furthermore, GAPS can explore hidden-sector DM, such as dark photon models that also evade direct and collider searches, via the hadronic decay of massive mediators $[23]$.

\subsection{Antihelium Sensitivity}

In 2018, the AMS-02 collaboration reported eight antihelium nucleus candidate events. Full information on these tentative events has yet to be published, but there is broad consensus that the confirmation of cosmic antihelium nuclei would imply new physics. Various models attempt to explain the potential antihelium detection by AMS-02. These models include standard cosmic rays with heavily-tuned formation models ${ }^{[24][25][26]}$, new DM annihilation channels ${ }^{[27]}$, and even the existence of an antistar within our Galaxy[28][29]. The projected antihelium nuclei fluxes in the GAPS energy range could span many orders of magnitude. Fig. 5 illustrates examples of predicted antihelium nuclei fluxes for dark matter with various masses annihilating into different channels, overlaid with the predicted astrophysical antihelium flux. Three GAPS LDB flights will provide the strongest sensitivity to low-energy antihelium-3 nuclei, with the potential to probe dark matter models annihilating into $\mathrm{W}^{+} \mathrm{W}^{-}{ }^{[12]}$ (also see more details in A. Stoessl's contribution).

Furthermore, the AMS-02 events shown at conferences have momenta of $>10 \mathrm{GeV} / c$, while GAPS is sensitive to the low-energy range $(\leq 0.25 \mathrm{GeV} / n)$ where the contribution from new physics is 


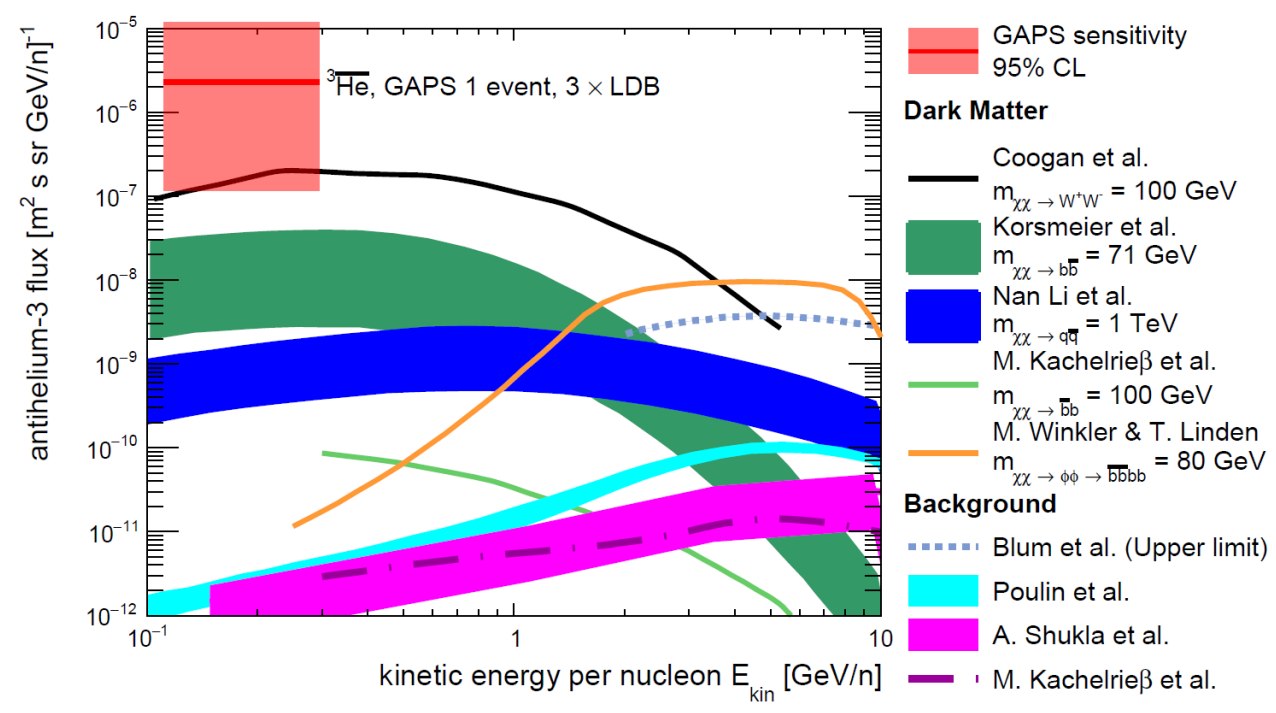

Figure 5: GAPS antihelium-3 nuclei sensitivity ${ }^{[12]}$ (95\% C.L.) for three LDB flights in comparison with predictions from a variety of DM and standard astrophysical background models. The uncertainties in the sensitivities are estimated using the upper and lower limits of true antihelium-3 nuclei detection from the $95 \%$ confidence interval, based on the calculated mean number of background event.

expected to be highest in most models. Using a completely different antinuclei detection technique from AMS-02, GAPS is the only experiment in the near future that can independently test the existence of low-energy cosmic antihelium nuclei.

\section{Conclusions}

The GAPS (General Antiparticle Spectrometer) Antarctic long-duration balloon program is the first experiment optimized for low-energy $(\lessgtr 0.25 \mathrm{GeV} / n)$ cosmic antinuclei. In the coming decade, the GAPS experiment will:

- deliver a first-time dedicated search for low-energy antideuterons, an unambiguous signal of new physics that probes a wide array of DM models, or improve limits by two orders of magnitude, significantly constraining current viable DM scenarios;

- provide a precision antiproton spectrum in an unexplored low-energy region, permitting leading constraints on $<10 \mathrm{GeV} \mathrm{DM}$, the best limits on primordial black hole evaporation on Galactic length scales, and novel insight on cosmic-ray propagation models; and

- open sensitivity to low-energy cosmic antihelium, in particular to investigate the candidate antihelium events reported by AMS-02.

The GAPS design has nearly completed, and the construction and integration has begun. The instrument is on schedule for the initial Antarctic flight in late 2022.

\section{Acknowledgment}

This work is supported in the U.S. by NASA APRA grants (NNX17AB44G, NNX17AB45G, NNX17AB46G, and NNX17AB47G) and in Japan by JAXA/ISAS Small Science Program FY2017. 
P. von Doetinchem received support from the National Science Foundation under award PHY1551980. H. Fuke is supported by JSPS KAKENHI grants (JP17H01136 and JP19H05198) and Mitsubishi Foundation Research Grant 2019-10038. K. Perez and M. Xiao are supported by HeisingSimons award 2018-0766. F. Rogers is supported through the National Science Foundation Graduate Research Fellowship under Grant No. 1122374. Y. Shimizu receives support from JSPS KAKENHI grant JP20K04002 and Sumitomo Foundation Grant No. 180322. This work is supported in Italy by Istituto Nazionale di Fisica Nucleare (INFN) and by the Italian Space Agency through the ASI INFN agreement no. 2018-28-HH.0: "Partecipazione italiana al GAPS - General AntiParticle Spectrometer". The technical support and advanced computing resources from the University of Hawaii Information Technology Services - Cyberinfrastructure are gratefully acknowledged.

\section{References}

[1] P. Von. Doetinchem, K Perez, et al., JACP 08 (2020) 035.

[2] T. Aramaki et al., Astropart. Phys. 74 (2016) 6-13.

[3] H. Fuke, H et al. Phys. Rev. Lett. 95, 081101 (2005).

[4] M. Korsmeier, F. Donato, \& N. Fornengo, Phys. Rev. D 97 (2018) 103011.

[5] T. Aramaki et al., Astropart. Phys. 49 (2013) 52-62.

[6] K. Perez et al., NIMA 905 (2018) 12.

[7] M. Kozai et al., NIMA 947 (2019) 162695.

[8] F. Rogers et al., JINST 14 (10) (2019) P10009.

[9] H. Fuke et al., Trans. JSASS Aerospace Tech. Japan Vol.14 No.ists30 (2016).

[10] S. Agostinelli et al., NIMA 506 (2003) 250.

[11] J. Allison et al., IEEE Transactions on Nuclear Science 53 (2006) 270.

[12] N. Saffold et al. Astro.Part.Phys. 130 (2021) 102580

[13] G. Arcadi, F. S. Queiroz and C. Siqueira, PLB 775 (2017) 196.

[14] K. Abe et al., Phys. Rev. Lett. 108 (2012) 051102.

[15] O. Adriani et al., Phys. Rev. Lett. 105 (2010) 121101.

[16] H. Baer and S. Profumo, JCAP 0512 (2005) 008.

[17] L. Dal and A. Raklev, Phys. Rev. D 89 (2014) 103504.

[18] Y. Cui, J. D. Mason, \& L. Randall, JHEP 017 (2010) 1011.

[19] D. Cerde no, M. Peir o and S. Robles, JCAP 1408 (2014) 005.

[20] N. Fornengo, L. Maccione and A. Vittino, JCAP 1309 (2013) 031.

[21] C. B. Br'auninger, \& M. Cirelli, Phys. Lett. B 678 (2009) 20-31.

[22] A. Hryczuk, I. Cholis, R. Iengo, M. Tavakoli and P. Ullio, JCAP 1407 (2014) 031.

[23] L. Randall, \& W. L. Xu, JHEP 81 (2020) 2020.

[24] K. Blum et al., Phys. Rev. D 96 (2017) 103021.

[25] A. Coogan and S. Profumo, Phys. Rev. D 96 (2017) 083020.

[26] Y-C. Ding et al., JCAP 06 (2019) 004.

[27] M. W. Winkler and T. Linden, Phys. Rev. Lett. 126 (2021) 101101.

[28] V. Poulin et al., Phys. Rev. D 99 (2019) 023016.

[29] K. M. Belotsky et al., Physics of Atomic Nuclei 63 (2000) 233. 


\section{Full Authors List: GAPS Collaboration}

T. Aramaki ${ }^{1}$, R. Bird ${ }^{2}$, M. Boezio ${ }^{3,4}$, S. E. Boggs ${ }^{5}$, V. Bonvicini ${ }^{3}$, D. Campana ${ }^{6}$, W. W. Craig ${ }^{7}$, E. Everson ${ }^{2}$, L. Fabris ${ }^{8}$, H. Fuke ${ }^{9}$, F. Gahbauer ${ }^{10}$, I. Garcia ${ }^{2}$, C. Gerrity ${ }^{11}$, C. J. Hailey ${ }^{10}$, T. Hayashi ${ }^{2}$, C. Kato ${ }^{12}$, A. Kawachi ${ }^{13}$, S. Kobayashi ${ }^{13}$, M. Kozai ${ }^{9}$, A. Lenni ${ }^{3,14}$, A. Lowell ${ }^{7}$, M. Manghisoni ${ }^{15,16}$, N. Marcelli ${ }^{17,18}$, B. Mochizuki ${ }^{7}$, S. A. I. Mognet ${ }^{19}$, K. Munakata $^{12}$, R. Munini ${ }^{3,4}$, Y. Nakagami ${ }^{20}$, J. Olson ${ }^{21}$, R. A. Ong ${ }^{2}$, G. Osteria ${ }^{6}$, K. Perez ${ }^{22}$, S. Quinn ${ }^{2}$, V. Re ${ }^{15,16}$, E. Riceputi ${ }^{15,16}$, B. Roach ${ }^{22}$, F. Rogers ${ }^{22}$, J. A. Ryan ${ }^{2}$, N. Saffold ${ }^{10}$, V. Scotti ${ }^{6,23}$, Y. Shimizu ${ }^{24}$, M. Sonzogni ${ }^{15,16}$, R. Sparvoli ${ }^{17,18}$, A. Stoess ${ }^{11}$, A. Tiberio ${ }^{25}$, E. Vannuccini ${ }^{25}$, P. von Doetinchem ${ }^{11}$, T. Wada ${ }^{20}$, M. Xiao ${ }^{22}$, M. Yamatami ${ }^{9}$, A. Yoshida ${ }^{20}$, T. Yoshida ${ }^{9}$, G. Zampa ${ }^{3}$, and J. Zweerink ${ }^{2}$

${ }^{1}$ Northeastern University, 360 Huntington Avenue, Boston, MA 02115, USA.

${ }^{2}$ University of California, Los Angeles, Los Angeles, CA 90095, USA.

${ }^{3}$ INFN, Sezione di Trieste, I-34149 Trieste, Italy.

${ }^{4}$ IFPU, I-34014 Trieste, Italy.

${ }^{5}$ University of California, San Diego, La Jolla, CA 90037, USA.

${ }^{6}$ INFN, Sezione di Napoli, I-80126 Naples, Italy.

${ }^{7}$ Space Sciences Laboratory, University of California, Berkeley, 7 Gauss Way, Berkeley, CA 94720 , USA.

${ }^{8}$ Oak Ridge National Laboratory, Oak Ridge, TN 37831, USA.

${ }^{9}$ Institute of Space and Astronautical Science, Japan Aerospace Exploration Agency (ISAS/JAXA), Sagamihara, Kanagawa 252-5210, Japan.

${ }^{10}$ Columbia University, New York, NY 10027, USA.

${ }^{11}$ University of Hawaii at Manoa, Honolulu, HI 96822 USA.

${ }^{12}$ Shinshu University, Matsumoto, Nagano 390-8621, Japan.

${ }^{13}$ Tokai University, Hiratsuka, Kanagawa 259-1292, Japan.

${ }^{14}$ Universitá di Trieste, I-34127 Trieste, Italy.

${ }^{15}$ INFN, Sezione di Pavia, I-27100 Pavia, Italy.

${ }^{16}$ Universitá di Bergamo, I-24044 Dalmine (BG), Italy.

${ }^{17}$ INFN, Sezione di Rome "Tor Vergata", I-00133 Rome, Italy.

${ }^{18}$ Universitá di Roma "Tor Vergata", I-00133 Rome, Italy.

${ }^{19}$ Pennsylvania State University, University Park, PA 16802 USA.

${ }^{20}$ Aoyama Gakuin University, Sagamihara, Kanagawa 252-5258, Japan.

${ }^{21}$ Heliospace Corporation, Berkeley, CA 94710, USA.

${ }^{22}$ Massachusetts Institute of Technology, Cambridge, MA 02139, USA.

${ }^{23}$ Universitá di Napoli "Federico II", I-80138 Naples, Italy.

${ }^{24}$ Kanagawa University, Yokohama, Kanagawa 221-8686, Japan.

${ }^{25}$ INFN, Sezione di Firenze, I-50019 Sesto Fiorentino, Florence, Italy. 